\title{
Negative Poisson's ratios in siliceous zeolite MFI-silicalite
}

\author{
Carmen Sanchez-Valle, ${ }^{1,2, a)}$ Zoe A. D. Lethbridge, ${ }^{3, a}$ Stanislav V. Sinogeikin,, ${ }^{1, b}$ \\ Jennifer J. Williams, ${ }^{4}$ Richard I. Walton, ${ }^{3}$ Kenneth E. Evans, ${ }^{4}$ and Jay D. Bass ${ }^{2}$ \\ ${ }_{1}^{1}$ Institute for Mineralogy and Petrology, ETH Zurich, CH-8092 Zurich, Switzerland \\ ${ }^{2}$ Department of Geology, University of Illinois, Urbana, Illinois 61801, USA \\ ${ }^{3}$ Department of Chemistry, University of Warwick, Gibbet Hill Road, Coventry CV4 7AL, \\ United Kingdom \\ ${ }^{4}$ School of Engineering, Computer Science and Mathematics, University of Exeter, \\ Exeter EX4 4QF, United Kingdom
}

(Received 28 January 2008; accepted 27 March 2008; published online 9 May 2008)

\begin{abstract}
Brillouin scattering measurements of the single-crystal elastic properties of the as-made zeolite silicalite $\left|\left(\mathrm{C}_{3} \mathrm{H}_{7}\right)_{4} \mathrm{NF}\right|_{4}\left[\mathrm{Si}_{96} \mathrm{O}_{192}\right]$-MFI provides the first experimental evidence for on-axis negative Poisson's ratios (auxeticity) in a synthetic zeolite structure. MFI laterally contracts when compressed and laterally expands when stretched along $x_{1}$ and $x_{2}$ directions in the (001) plane $\left(\nu_{12}=-0.061, \nu_{21}=-0.051\right)$. The aggregate Poisson's ratio of MFI, although positive, has an anomalously low value $\nu=0.175(3)$ compared to other silicate materials. These results suggest that the template-free MFI-silicalite $\left[\mathrm{Si}_{96} \mathrm{O}_{192}\right]$ might have potential applications as tunable sieve where molecular discriminating characteristics are adjusted by application of stress along specific axes. (C) 2008 American Institute of Physics. [DOI: 10.1063/1.2912061]
\end{abstract}

Materials with a negative Poisson's ratio known as auxetics are solids that exhibit the unusual property of laterally expanding under uniaxial tension and laterally shrinking under uniaxial compression. ${ }^{1,2}$ Far from being a mere scientific curiosity, these materials have important applications in various technologies and are of interest to a range of scientific communities, from chemistry to material engineering. Their unusual mechanical properties, indeed, give rise to a long list of measured and predicted technological enhancements, such as improved plane-strain fracture toughness, and increased indentation resistance, ${ }^{2}$ improved honeycomb dielectric properties, ${ }^{3}$ self-adaptative vibrational damping properties, ${ }^{4}$ increased shear modulus, improved sound and shock absorption, and the ability to form dome-shaped surfaces. ${ }^{5}$ The identification of auxetic behavior on an atomic level has also opened up the possibility of molecular-scale amplifiers and other nanoscale devices. ${ }^{6}$

Given the widespread potential applications of these materials, experimental and theoretical investigations have focused for the past few years into the identification and refinement of new auxetic materials and understanding the physical basis of this unusual property. Negative Poisson's ratios have been experimentally identified in a number of organic and inorganic materials, both in crystalline and amorphous forms, including polymers, foams, elemental cubic metals and alloys, and the silica polymorph $\alpha$-cristobalite. ${ }^{5-12}$ The latter is, in fact, the only known crystalline silicate possessing an aggregate negative Poisson's ratio $(\nu=-0.16)$, which has been interpreted as arising from the concurrent rotation of the $\mathrm{SiO}_{4}$-tetrahedral building

\footnotetext{
${ }^{\text {a) }}$ Authors to whom correspondence should be addressed. Electronic addresses: carmen.sanchez@erdw.ethz.ch and z.lethbridge@warwick.ac.uk.

${ }^{b)}$ Present address: HPCAT-APS, Carnegie Institution of Washington, Argonne, IL, USA.
}

units $^{13,14}$ under an applied stress. The search for auxetic materials has targeted other related low-density openframework $\mathrm{SiO}_{2}$-based structures, and recent simulations performed by Grima et al. have predicted negative Poisson's ratios in a number of idealized siliceous zeolites. ${ }^{15}$ However, the lack of accurate experimental data on the elastic properties of zeolites has precluded confirmation of these predictions for many years.

To address this problem, we recently applied Brillouin scattering spectroscopy and micromechanical techniques ${ }^{16,17}$ to investigate the single-crystal elastic properties of the naturally occurring aluminosilicate zeolite natrolite (NAT structure type). Calculations had suggested that the hypothetical all-silica NAT zeolite framework is auxetic. ${ }^{15}$ Measurements of the acoustic velocities on natural specimens of natrolite provided the full set of individual $C_{i j}$, aggregate elastic moduli, and directional Poisson's ratios at ambient conditions. ${ }^{16}$ Although on-axis $\nu_{i j}$ and aggregate Poisson's ratios $\nu$ were found to be all positive, further off-axis analysis revealed negative Poisson's ratios when stress is applied at $45^{\circ}$ to the principal crystallographic axis in the (001) plane. ${ }^{18,19}$ The identification of auxetic zeolite networks indicates that their capacity for adsorption, diffusion, or reaction may be modified by the application of strain along specific directions. ${ }^{15,20,21}$ We have now extended the search for auxetic behavior to synthetic zeolites that can be specially tailored for particular industrial applications.

In this paper, we present results for the elastic properties of the synthetic silicalite zeolite, ${ }^{22}$ a pure silica form of the aluminosilicate ZSM-5 zeolite (MFI structure type) that is industrially used in the production of para-xylene ${ }^{23}$ and the conversion of methanol to gasoline. ${ }^{24}$ In the synthesis procedure, the tetrapropylammonium (TPA) cation can be used as a "template" to direct the formation of silicalite and is found 
within the silicate framework of the "as-made" material. This zeolite has two channel systems: straight channels, which run along the $x_{2}$ axis, and sinusoidal channels along $x_{1}$; in the as-made form of silicalite, the TPA cations are located at the intersections of these two channels. ${ }^{25}$ The elastic properties of synthetic zeolites have so far eluded direct measurement, due in part to the difficulties in obtaining sufficiently large crystals. ${ }^{26}$ Synthetic zeolites generally form as microcrystalline powders; however, to perform Brillouin spectroscopy, crystals of around $80-100 \mu \mathrm{m}$ are required. Here, we have utilized the "bulk-material dissolution" technique ${ }^{27}$ to synthesize large crystals of MFI-silicalite and to report the first determination of the full set of elastic constants of as-made silicalite by Brillouin scattering spectroscopy. Analysis of the elastic data shows that the on-axis Poisson's ratios $\nu_{i j}$ have negative values in the (001) plane when loaded along the $x_{1}$ and $x_{2}$ directions, providing the first experimental evidence for on-axis negative Poisson's ratios in a zeolite structure.

The silicalite single crystals used in this study were hydrothermally synthesized by using a piece of quartz tube (approximately $5 \times 10 \mathrm{~mm}^{2}$ ), hydrofluoric acid $(40 \%)$, and TPA hydroxide solution $(25 \%)$. The products are made up of clear prismatic crystals elongated along the $c$ axis ( $\sim 500 \mu \mathrm{m}$ in length) with square pyramidal caps on both ends. The sample was shown to be phase pure by powder $\mathrm{x}$-ray diffraction. The orthorhombic symmetry of silicalite, space group Pmna, ${ }^{28}$ was verified by using single-crystal x-ray diffraction, from which unit-cell parameters of $a$ $=20.019(5) \AA, b=19.963(9) \AA$, and $c=13.388(5) \AA$ and $V$ $=5350.4(6) \AA^{3}$ were obtained. As-synthesized TPAcontaining silicalite was determined to have the formula $\left|\left(\mathrm{C}_{3} \mathrm{H}_{7}\right)_{4} \mathrm{NF}\right|_{4}\left[\mathrm{Si}_{96} \mathrm{O}_{192}\right]$ by thermogravimetric and carbon, hydrogen, and nitrogen elemental analyses. Combining the chemical results with the crystallographic data gives a calculated density of $2.045(5) \mathrm{g} \mathrm{cm}^{-3}$. Three crystals of high optical quality were preoriented by using both crystal morphology and x-ray diffraction and then polished into 50-30 $\mu \mathrm{m}$ thick plates with parallel faces $\left[(150-250) \times 130 \mu \mathrm{m}^{2}\right.$ lateral dimensions]. Samples were oriented with a four-circle x-ray diffractometer before the Brillouin experiments. Although twinning is commonly found in silicalite crystals, ${ }^{28-30}$ no evidence of twins was indicated by the $\mathrm{x}$-ray in polished crystals used in our measurements.

Brillouin scattering measurements were performed by using an Ar ion laser $\left(\lambda_{0}=514.5 \mathrm{~nm}\right)$ as a light source and a six-pass tandem Fabry-Pérot interferometer to analyze the frequency of the scattered light. Details of the experimental setup are given elsewhere. ${ }^{31}$ All spectra were collected by using symmetric (platelet) scattering geometry with an angle of $90^{\circ}(\theta)$ between the incident and scattered lights. In this geometry, the sound velocities $\left(V_{i}\right)$ are related to the measured Brillouin frequency shifts $\Delta \omega_{i}$ according to $V_{i}$ $=\Delta \omega_{i} \lambda_{0} / 2 \sin (\theta / 2){ }^{32}$ The Brillouin system was calibrated prior to the experiments by using a $\mathrm{MgO}$ single-crystal standard to reduce geometric or other possible systematic errors in velocity determinations. The reproducibility in repeated measurements was found to be better than $0.5 \%$ of the measured velocities. A representative Brillouin spectrum of silicalite is shown in Fig. 1.

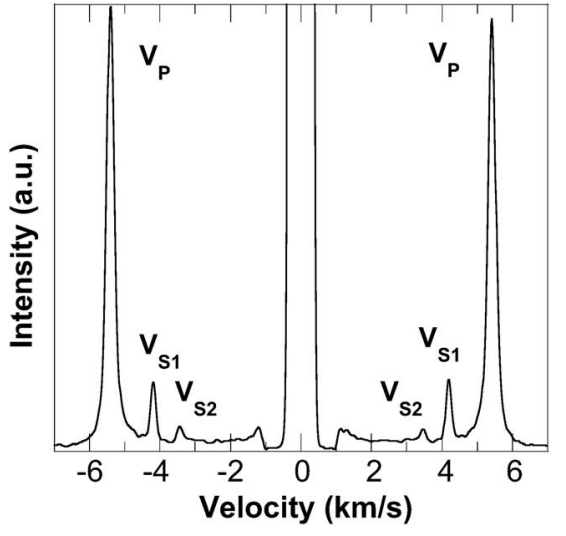

FIG. 1. Representative Brillouin spectrum of silicalite collected at ambient conditions in the $[0.5000,-0.7367,0.4553]$ direction within the $(0.006,-0.5286,-0.8488)$ crystallographic plane. The compressional $\left(V_{P}\right)$ and the two shear $\left(V_{S 1}\right.$ and $\left.V_{S 2}\right)$ acoustic modes are labeled. The features at $\pm 1 \mathrm{~km} / \mathrm{s}$ are unattenuated tails of the Rayleigh peak

Sound velocity measurements were performed in each sample plate over an angular range of $180^{\circ}$ in $15^{\circ}$ intervals from both sides of the plates. All spectra showed the compressional $\left(V_{P}\right)$ and at least one shear acoustic mode $\left(V_{S}\right)$. Two transverse modes were not observed in all crystallographic directions (Fig. 2). The final dataset consisted of 166 acoustic modes measured in 29 crystallographic directions, with redundant measurement along the principal axes [100] and [001] performed on at least two different samples (Fig. 2).

The nine independent single-crystal elastic constants $C_{i j}$ of silicalite were determined by using a least-square fitting routine that minimizes the differences between measured and calculated velocities through iterative solution of Christoffel's equations. ${ }^{33}$ Inversions were performed with different initial sets of $C_{i j}$ stiffness moduli to verify the independence of the results from the starting model. The best-fit elastic constant model gives a root mean square value of $0.026 \mathrm{~km} / \mathrm{s}$ with respect to the observed velocities, providing a precision better than $2 \%$ in most of the calculated elastic constants (Table I). The full data set collected from the sample plates is displayed in Fig. 2 together with the calculated velocity model from the inversion results. The best-fit models of the elastic stiffness $\left(C_{i j}\right)$ and compliance $S_{i j}\left(C_{i j}{ }^{-1}\right)$ matrices, and the aggregate elastic properties of silicalite calculated from $C_{i j}$ by using the Voigt-Reuss-Hill averaging schemes, ${ }^{34}$ are summarized in Table I.

The relatively high shear modulus of silicalite $[G$ $=26.3(5) \mathrm{GPa}]$ compared to the bulk modulus $\left[K_{s}\right.$ $=31.7(6) \mathrm{GPa}]$ indicates that the structure is difficult to deform under shear stress but easy to deform volumetrically. The aggregate Poisson's ratio, calculated as $\nu=\left(3 K_{s}\right.$ $-2 G) /\left(6 K_{s}+2 G\right)$, consequently takes a value $\nu=0.175(3)$, which is exceptionally low compared to typical values found in most crystalline compounds $(0.20-0.27){ }^{35}$ This result anticipates the anomalous elastic behavior of silicalite, as discussed below.

To gain further insight into the elastic behavior of silicalite, we examine Young's moduli and Poisson's ratio as a function of the specific directions of the applied stress and 


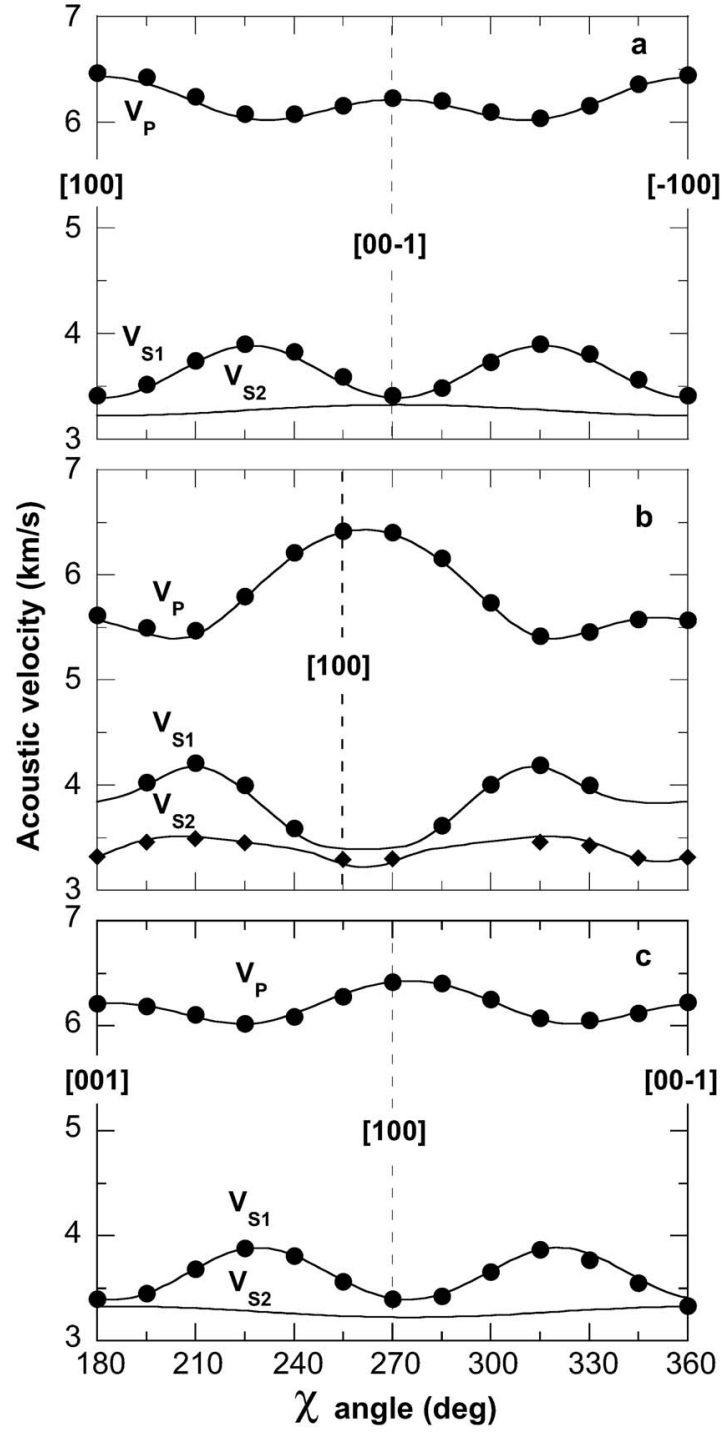

FIG. 2. Measured velocities in silicalite as a function of the angle in (a) $(-0.0217,-0.9994,-0.0265), \quad$ (b) $(0.006,-0.5286,-0.8488)$, and (c) $(0.0229,-0.9993,-0.0308)$ crystallographic planes. Solid lines are calculated from the best-fit $C_{i j}$ model. Error bars are smaller than the symbol size.

strain. The directional Young's moduli $E_{i}$ for loading in the $x_{i}$ $(i=1,2,3)$ direction and Poisson's ratios $\nu_{i j}$ in the $x_{i} x_{j}$ planes $(i, j=1,2,3)$ for loading in the $x_{i}$ direction are computed by using the $S_{i j}$ compliance constants (Table I) through the relationships:

TABLE I. Single-crystal stiffness $C_{i j}$, compliance $S_{i j}$ constants, and aggregate elastic properties (Voigt-Reuss-Hill averages) of silicalite. Numbers in parentheses indicate the errors on the last significant digit.

\begin{tabular}{clccc}
\hline \hline$i j$ & $C_{i j}(\mathrm{GPa})$ & $S_{i j}\left(10^{3} \mathrm{GPa}^{-1}\right)$ & Parameter & Value \\
\hline 11 & $84.5(8)$ & $12.6(3)$ & $\rho\left(\mathrm{g} / \mathrm{cm}^{3}\right)$ & $2.045(5)$ \\
22 & $68.2(1.2)$ & $15.0(3)$ & $K_{S}(\mathrm{GPa})$ & $31.7(6)$ \\
33 & $79.0(8)$ & $13.8(3)$ & $G(\mathrm{GPa})$ & $26.3(5)$ \\
44 & $22.6(2)$ & $44.2(9)$ & $V_{P}(\mathrm{~km} / \mathrm{s})$ & $5.71(8)$ \\
55 & $23.5(4)$ & $42.5(9)$ & $V_{S}(\mathrm{~km} / \mathrm{s})$ & $3.59(6)$ \\
66 & $21.2(2)$ & $47.2(1.0)$ & $V_{P} / V_{S}$ & 1.59 \\
12 & $-1.52(2)$ & $0.77(3)$ & $\nu$ & $0.175(3)$ \\
13 & $19.9(2)$ & $-3.28(7)$ & $E(\mathrm{GPa})$ & $61.8(1.2)$ \\
23 & $10.3(3)$ & $-2.15(5)$ & & \\
\hline \hline
\end{tabular}

TABLE II. Directional Poisson's ratios and Young moduli of silicalite. Errors are smaller than $3 \%$.

\begin{tabular}{lcc}
\hline \hline Orientation & Parameter & Value \\
\hline (100) plane & $\nu_{23}$ & 0.143 \\
& $\nu_{32}$ & 0.155 \\
(010) plane & $\nu_{13}$ & 0.260 \\
& $\nu_{31}$ & 0.237 \\
(001) plane & $\nu_{12}$ & -0.061 \\
& $\nu_{21}$ & -0.051 \\
[100] direction & $E_{1}(\mathrm{GPa})$ & 79.6 \\
{$[010]$ direction } & $E_{2}(\mathrm{GPa})$ & 66.7 \\
{$[001]$ direction } & $E_{3}(\mathrm{GPa})$ & 72.5 \\
\hline \hline & & \\
$E_{i}=1 / S_{i i}$, & &
\end{tabular}

The calculated on-axis $E_{i}$ and $\nu_{i j}$ are listed in Table II. The values taken by Young's modulus $E_{i}$ along the principal axis indicate that the linear compressibility of silicalite is slightly anisotropic, with the [100] axis being the stiffest and the [010] axis being the softest. A previous mechanical study of silicalite crystals has been reported by Wang et al. ${ }^{36}$ however, the values reported there $(E=3-4 \mathrm{GPa})$ are much lower than those obtained in this study and lower than might be expected on comparison with similar materials; for instance, the silica polymorph $\alpha$-cristobalite has $E_{1}=E_{2}$ $=59 \mathrm{GPa}$ and $E_{3}=42 \mathrm{GPa}$ (calculated from Ref. 12), and Brillouin spectroscopy studies of the cubic silica clathrate dodecasil $3 C,{ }^{37}$ which contains $\mathrm{N}_{2}, \mathrm{Ar}$, and $\mathrm{N}\left(\mathrm{CH}_{3}\right)_{3}$, gave a directional Young's modulus of $52 \mathrm{GPa}$.

The values obtained for as-made silicalite can be compared to the available elasticity data on other zeolites and related materials. We previously reported the elastic constants for the aluminosilicate zeolite natrolite. ${ }^{16}$ Young's modulus values are of similar magnitude, although the structural anisotropy in natrolite is highlighted by a higher value along the [001] axis $\left(E_{3}=111.1 \mathrm{GPa}\right)$ along which chains of linked clusters of five tetrahedra run. Lower values are found in the (001) plane $\left(E_{1}=57.2 \mathrm{GPa}, E_{2}=59.2 \mathrm{GPa}\right)$, where the structure is more open. The smaller spread of $E$ values in silicalite suggests less anisotropy in the tetrahedral connectivity. The clathrasil dodecasil 3C (MTN framework type) is the most closely related silicate whose elastic constants have been measured, since it also contains organic molecules from the synthesis procedure. ${ }^{35}$ However, MTN is purely a cage structure, in contrast to MFI which contains two channel systems.

The directional dependence of Poisson's ratio indicates prominent anisotropy (Table II). Over the principal directions, it takes values that range from +0.260 to -0.061 , even though the aggregate value remains positive $[\nu=0.175(3)]$. Negative values of Poisson's ratios are obtained in the (001) crystallographic plane when silicalite is loaded along the $x_{1}$ and $x_{2}$ directions. This behavior is a result of the positive value of the $S_{12}$ compliance coefficient, indicating that when compressed along the $x_{2}$ axis, the structure contracts along the $x_{1}$ axis (and vice versa) $\left(\nu_{12}=-0.061, \nu_{21}=-0.051\right)$. 


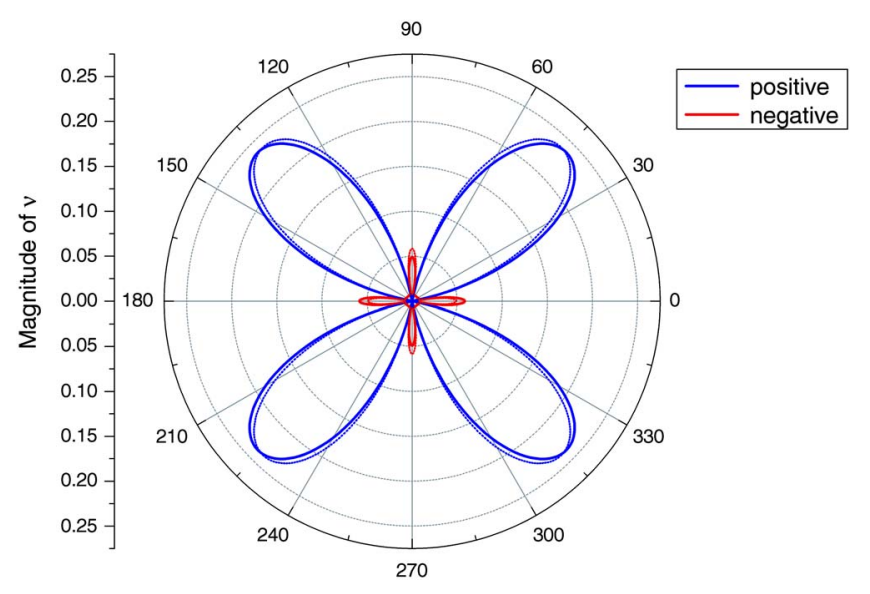

FIG. 3. (Color online) Off-axis analysis of the experimentally determined Poisson's ratios in the (001) plane of silicalite under rotation about the $x_{3}$ axis. Negative Poisson's ratios (red) are exhibited along the $x_{1}$ and $x_{2}$ crystallographic axes.

Given the presence of space-filling organic template molecules in the sample, it is difficult to identify the structural origin of this behavior. Elastic behavior in directions not coincident with the principal crystallographic directions was examined by applying transformation equations to the stiffness matrix. ${ }^{16,38}$ Plots of this off-axis analysis are shown in Fig. 3, where it can be seen that $\nu_{12}$ and $\nu_{21}$ are the minimum values reached by Poisson's ratio in the (001) plane, i.e.; the minimum values occur on axis. Examination of similar offaxis plots of (010) and (100) (not shown) indicates that Poisson's ratio is always positive in these planes. The magnitude of the auxeticity in MFI is, however, appreciably lower compared to that of other auxetic crystalline silicates; for instance, $\alpha$-cristobalite and natrolite have minimum $\nu$ values of -0.5 and -0.12 , respectively. This suggests that the presence of the TPA template occupying most of the free-pore space might restrict the rotation of the tetrahedral framework units, hence, reducing the flexibility of the structure and the auxetic effect. ${ }^{15,19}$ Attempts to remove the extra-framework template by either calcination or chemical means resulted in severe cracking of the crystals, presumably due to mechanical stress upon template loss, rendering them unsuitable for further Brillouin scattering measurements.

The experimental identification of auxetic behavior in MFI-silicalite is of interest following previous calculations of these properties. ${ }^{15}$ Negative Poisson's ratios were predicted in the (100) and (010) crystallographic planes for calcinated silicalite (in which the TPA cations have been removed), ${ }^{22}$ while the as-made samples have now been shown to exhibit auxetic behavior in (001). This observation emphasizes the dramatic effect of the organic template on the elastic properties of the silicalite framework and must be considered when understanding the flexibility of the structure. In the absence of further structural information, molecular simulations might provide a means to analyze possible structural deformations causing the anisotropy in elastic behavior and the negative Poisson's ratios observed in certain crystallographic directions; this is something we will investigate in the future.
In conclusion, measurements of the single-crystal elastic properties of MFI-silicalite by using Brillouin scattering provide the first direct experimental evidence for on-axis auxetic behavior in a synthetic zeolite structure. Maximum auxeticity is observed when the structure is loaded along the $x_{1}$ and $x_{2}$ axes in the (001) crystallographic plane $\left(\nu_{12}=-0.061\right.$, $\left.\nu_{21}=-0.051\right)$. This finding increases the number of crystalline structures that exhibits this counterintuitive elastic behavior, limited up to now to a few materials such as $\alpha-\mathrm{TeO}_{2}$ (paratellurite), some cubic elemental metals and alloys, and the uncommon minerals, $\alpha$-cristobalite and natrolite. The identification of auxetic behavior in MFI-silicalite suggests that the capacity for adsorption, diffusion, or reaction of the template-free material could be modified by the application of strain along specific axes, opening up the possibility of using this zeolite as a selective molecular sieve in ion exchange and catalytic technologies.

This work was supported by the USA National Science Foundation (Nos. EAR 0003383 and EAR0135642 to J.D.B.) and the Engineering and Physical Sciences Research Council, UK (No. EP/C516591/2). We thank C. W. Smith (University of Exeter) for useful discussions.

${ }^{1}$ K. E. Evans, M. A. Nkansah, I. J. Hutchinson, and S. C. Rogers, Nature (London) 353, 124 (1991).

${ }^{2}$ K. E. Evans and A. Alderson, Adv. Mater. (Weinheim, Ger.) 12, 617 (2000).

${ }^{3}$ F. C. Smith, F. Scarpa, and B. Chambers, IEEE Microw. Guid. Wave Lett. 10, 451 (2000).

${ }^{4}$ G. R. Tomlinson, J. Mech. Eng. Sci. 214, 103 (2001).

${ }^{5}$ R. S. Lakes, Science 235, 1038 (1987).

${ }^{6}$ R. H. Baughman, Nature (London) 425, 667 (2003).

${ }^{7}$ K. L. Alderson and K. E. Evans, J. Mater. Sci. 28, 4092 (1993).

${ }^{8}$ A. P. Pickles, K. L. Alderson, and K. E. Evans, Polym. Eng. Sci. 36, 636 (1996).

${ }^{9}$ K. E. Evans, M. A. Nkansah, and I. J. Hutchinson, Acta Metall. Mater. 42, 1289 (1994).

${ }^{10}$ C. W. Smith, J. N. Grima, and K. E. Evans, Acta Mater. 48, 4349 (2000).

${ }^{11}$ R. H. Baughman, J. M. Shacklette, A. A. Zakhidov, and S. Strafström, Nature (London) 392, 362 (1998).

${ }^{12}$ A. Yeganeh-Haeri, D. J. Weidner, and J. B. Parise, Science 257, 650 (1992).

${ }^{13}$ N. R. Keskar and J. R. Chelikowsky, Nature (London) 358, 222 (1992).

${ }^{14}$ A. Alderson and K. E. Evans, Phys. Rev. Lett. 89, 225503 (2002).

${ }^{15}$ J. N. Grima, R. Jackson, A. Alderson, and K. E. Evans, Adv. Mater. (Weinheim, Ger.) 12, 1912 (2000).

${ }^{16}$ C. Sanchez-Valle, S. V. Sinogeikin, Z. A. D. Lethbridge, C. W. Smith, K. E. Evans, and J. D. Bass, J. Appl. Phys. 98, 53508 (2005).

${ }^{17}$ Z. A. D. Lethbridge, J. J. Williams, R. I. Walton, C. W. Smith, R. M. Hooper, and K. E. Evans, Acta Mater. 54, 2533 (2006).

${ }^{18}$ J. N. Grima, R. Gatt, V. Zammit, J. J. Williams, K. E. Evans, A. Alderson, and R. I. Walton, J. Appl. Phys. 101, 086102 (2007).

${ }^{19}$ J. J. Williams, C. W. Smith, K. E. Evans, Z. A. D. Lethbridge, and R. I. Walton, Chem. Mater. 19, 2423 (2007).

${ }^{20}$ Y. Lee, J. A. Hriljac, T. Vogt, J. B. Parise, and G. Artioli, J. Am. Chem. Soc. 123, 12732 (2001).

${ }^{21}$ Y. Lee, T. Vogt, J. A. Hriljac, J. B. Parise, and G. Artioli, J. Am. Chem. Soc. 124, 5466 (2002).

${ }^{22}$ E. M. Flanigen, J. M. Bennett, R. W. Grose, J. P. Cohen, R. L. Patton, R. M. Kirchner, and J. V. Smith, Nature (London) 271, 512 (1978).

${ }^{23}$ N. Y. Chen, Ind. Eng. Chem. Res. 40, 4157 (2001).

${ }^{24}$ M. Stöcker, Microporous Mesoporous Mater. 29, 3 (1999).

${ }^{25}$ H. van Koningsveld, H. van Bekkum, and J. C. Jansen, Acta Crystallogr., Sect. B: Struct. Sci. 43, 127 (1987).

${ }^{26}$ Z. A. D. Lethbridge, J. J. Williams, R. I. Walton, K. E. Evans, and C. W. Smith, Microporous Mesoporous Mater. 79, 339 (2005).

${ }^{27}$ S. Shimizu and H. Hamada, Angew. Chem., Int. Ed. 38, 2725 (1999).

${ }^{28}$ G. D. Price, J. J. Pluth, J. V. Smith, J. M. Bennett, and R. L. Patton, J. 
Am. Chem. Soc. 104, 5971 (1982).

${ }^{29}$ C. Weidenthaler, R. X. Fischer, R. D. Shannon, and O. Medenbach, J. Phys. Chem. 98, 12687 (1994).

${ }^{30}$ O. Geier, S. Vasenkov, E. Lehmann, J. Karger, U. Schemmert, R. A. Rakoczy, and J. Weitkamp, J. Phys. Chem. B 105, 10217 (2001).

${ }^{31}$ S. V. Sinogeikin, T. Katsura, and J. D. Bass, J. Geophys. Res. 103, 20819 (1998).

${ }^{32}$ C. H. Whitfield, E. M. Brody, and W. A. Bassett, Rev. Sci. Instrum. 47, 942 (1976).
${ }^{33}$ D. J. Weidner and H. R. Carleton, J. Geophys. Res. 82, 1334 (1977).

${ }^{34}$ J. P. Watt, G. F. Davies, and R. J. O'Connell, Rev. Geophys. Space Phys. 14, 541 (1976).

${ }^{35}$ J. D. Bass, Mineral Physics and Crystallography: A Handbook of Physical Constants (AGU, Washington, DC, 1995).

${ }^{36}$ Z. Wang, R. F. Lobo, and J. Lambros, Microporous Mesoporous Mater. 57, 1 (2002)

${ }^{37}$ R. Freimann and H. Küppers, Phys. Status Solidi A 123, K123 (1991).

${ }^{38}$ R. F. S. Hearmon, Rev. Mod. Phys. 18, 409 (1946); Adv. Phys. 5, 323 (1956). 